\title{
Type D personality is associated with impaired health-related quality of life 7 years following heart transplantation
}

\author{
Susanne S. Pedersen ${ }^{\mathrm{a}, \mathrm{b}, *}$, Pieter G. Holkamp ${ }^{\mathrm{a}}$, Kadir Caliskan $^{\mathrm{a}}$, Ron T. van Domburga \\ Ruud A.M. Erdman ${ }^{\mathrm{a}, \mathrm{c}}$, Aggie H.M.M. Balk \\ a Department of Cardiology, Thoraxcentre, Erasmus Medical Centre, Rotterdam, The Netherlands \\ ${ }^{\mathrm{b}}$ Centre of Research on Psychology in Somatic diseases (CoRPS), Tilburg University, Tilburg, The Netherlands \\ ${ }^{\mathrm{c}}$ Department of Medical Psychology and Psychotherapy, Erasmus Medical Centre, Rotterdam, The Netherlands
}

Received 21 March 2006; received in revised form 25 May 2006; accepted 27 June 2006

\begin{abstract}
Objective: Health-related quality of life (HRQoL) following transplantation is gaining importance as an endpoint, but little is known about the role of normal personality traits as a determinant of HRQoL in this patient group. We investigated whether Type D personality (tendency to experience increased negative emotions paired with the nonexpression of these emotions) was associated with impaired HRQoL in heart transplant recipients. Methods: Data were collected from all surviving heart transplant recipients $\geq 21$ years of age $(n=186)$ with a mean (S.D.) of 7 (5) years following transplantation. Patients completed the Short-Form Health Survey 36 (SF-36) and the Type D Scale (DS14). Clinical data were obtained from the medical records. Results: Of the 186 patients, $18 \%$ had a Type D personality. Type D patients had significantly worse scores on the Physical Component scale (PCS)
\end{abstract}

$(P=.04)$ and the Mental Component scale (MCS) $(P<.001)$ of the SF-36 and all the SF-36 subdomains (all $P<.01$ ) compared with non-Type D patients, except for Bodily Pain. Type D personality remained an independent determinant of impaired PCS [odds ratio (OR), 3.62; 95\% confidence interval (CI), 1.25-10.45] and MCS (OR, 6.13; 95\% CI, 2.23-16.83) and six of the eight subscales of the SF-36, adjusting for demographic and clinical characteristics. Conclusions: Type D personality was associated with more than a three- to six-fold increased risk of impaired HRQoL in heart transplant recipients, showing that the Type $\mathrm{D}$ personality construct also has value in heart transplant recipients. The adoption of a personality approach may lead to improved risk stratification in research and clinical practice in this patient group.

(C) 2006 Elsevier Inc. All rights reserved.

Keywords: Health-related quality of life; Heart transplantation; Type D personality

\section{Introduction}

Since the survival rate for heart transplant recipients has improved to $60 \%$ at 7 years, health-related quality of life (HRQoL) following transplantation has become an important endpoint [1]. The study of HRQoL in this patient group is not only important because it may lead to improved patient-centered care [2], HRQoL has also been associated with increased risk of mortality in patients with cardiovas-

* Corresponding author. CoRPS, Department of Medical Psychology, Room P503a, Tilburg University, Warandelaan 2, PO Box 90153, 5000 LE Tilburg, The Netherlands. Tel.: +31 13466 2503; fax: +31 134662370 .

E-mail address: s.s.pedersen@uvt.nl (S.S. Pedersen). cular disease (CVD) [3-5]. In order to identify subgroups of recipients at risk for impaired $\mathrm{HRQoL}$ and to enhance secondary prevention in these high-risk patients, knowledge of the determinants of HRQoL is important. Knowledge of these determinants may also close the gap between research and clinical practice [2].

In this context, a personality approach may provide a particularly valuable framework, as personality has much explanatory power of individual differences in psychological distress, HRQoL, and adverse health outcomes [6,7]. From a screening point of view, a personality approach is also advantageous, since personality traits exert stable effects on behaviour, whereas mood states (e.g., anxiety and depression) are transient and fluctuate over time. A 
personality approach has already been used in the field of heart transplantation, and personality factors have been associated with posttransplant compliance, morbidity, and mortality [8-10]. Emphasis, however, has primarily been on personality disorders or on single, normal, personality traits, rather than the interaction of traits. In addition, few studies have investigated the impact of personality on HRQoL.

The distressed (Type D) personality is an emerging risk factor for adverse health outcomes, impaired HRQoL, fatigue, and several forms of distress, including anxiety, depression, and posttraumatic stress, in patients with CVD [6,11-16]. Type D is based on two normal, broad, and stable personality traits (i.e. negative affectivity and social inhibition), and is defined as the tendency to experience increased negative distress paired with the nonexpression of these emotions in social interactions [17]. The impact of Type D personality has not yet been investigated in the context of heart transplantation, although a derivation from the Millon Health Behavioral Inventory (i.e. the "prototypic high-distress patient") that resembles Type D has previously been associated with increased risk of mortality in heart transplant recipients [8].

The objective of the current study was to investigate whether Type D personality is an independent determinant of impaired HRQoL in this distinct patient group.

\section{Methods}

\section{Population and procedure}

In February 2003, all surviving heart transplant recipients $\geq 21$ years of age $(n=211)$ who had been transplanted in the Erasmus Medical Centre Rotterdam between 1985 and early 2003 were approached in writing and asked to complete a set of psychological questionnaires. A reminder was sent out in April 2003 to nonresponders. All patients provided written informed consent.

\section{Measures}

\section{Demographic and clinical characteristics}

Demographic factors (sex, age, marital status, education, and working status) were obtained through purposedesigned questions in the questionnaire. Clinical factors (time since transplantation, number of rejection episodes in the first year posttransplantation, the need for renal replacement therapy, the presence of malignancy, diabetes posttransplantation, and immunosuppressant medication) were obtained from the medical records.

\section{Type D personality}

Type D personality was assessed with the Type D Scale (DS14), a 14-item scale with 7 items measuring Negative Affectivity (NA) (e.g., "I often feel unhappy") and 7 items measuring Social Inhibition (SI) (e.g., "I am a closed kind of person") [17]. Items are answered on a 5-point Likert scale ranging from 0 (false) to 4 (true). A standardised cutoff $\geq 10$ on both subscales indicates Type D caseness [17]. The DS14 is a valid and reliable scale with Cronbach's $\alpha$ of .88 and .86 for the NA and SI subscales, respectively [17]. The test-retest correlations over a 3-month period were .82 and .72 for the SI and NA scales, respectively [17]. It is important to note that Type D reflects a normal personality type rather than psychopathology, and that it is not merely a measure of negative affect, such as depression, as it also takes into account how patients deal with this affect $[6,17]$.

\section{Health-related quality of life}

Perceived HRQoL was measured with the Short-Form Health Survey 36 (SF-36), a standardised instrument developed to measure functioning and well-being in primary care populations and in patients with chronic diseases [18]. The SF-36 comprises 36 items, divided into eight subscales: Physical Functioning, Role Physical Functioning, Social Functioning, Role Emotional Functioning, Mental Health, Vitality, Bodily Pain, and General Health. Scores on the subscales are linearly converted to a score between 0 and 100, with a higher score representing a better functioning, although for Bodily Pain a high score indicates less pain. The Dutch version of the SF-36 has been validated in the Dutch population and has a good internal consistency with a mean Cronbach's $\alpha$ of .84 across all scales [19]. Using an algorithm, the eight subscales can be summarised into two component summary scores, the Physical Component summary score (PCS) and the Mental Component summary score (MCS) [20].

Table 1

Patient characteristics stratified by Type D personality

\begin{tabular}{llll}
\hline & $\begin{array}{l}\text { Type D } \\
(n=34)\end{array}$ & $\begin{array}{l}\text { Non-Type D } \\
(n=152)\end{array}$ & $P$ \\
\hline Males (\%) & 79 & 73 & .58 \\
Age (years), mean (S.D.) & $48(12)$ & $50(10)$ & .32 \\
Time since transplant (years), & $8(4)$ & $7(5)$ & .41 \\
$\quad$ mean (S.D.) & & & \\
No partner (\%) & 38 & 15 & $<.01^{*}$ \\
Low education (\%) & 47 & 22 & $.01^{*}$ \\
Not working (\%) & 71 & 68 & .38 \\
2 or more rejection & 35 & 45 & .11 \\
$\quad$ episodes in the first & & & .96 \\
$\quad$ year posttransplantation (\%) & & & .57 \\
Need for renal replacement & 18 & 8 & .24 \\
$\quad$ therapy (\%) & & & \\
Presence of malignancy (\%) & 18 & 20 & \\
Diabetes posttransplantation (\%) & 16 & 22 & \\
Cyclosporine-based & 71 & 58 & \\
$\quad$ immunosuppressant therapy & \\
\hline$\quad$ a Versus tacrolimus-based immunosuppressant therapy. & & &
\end{tabular}




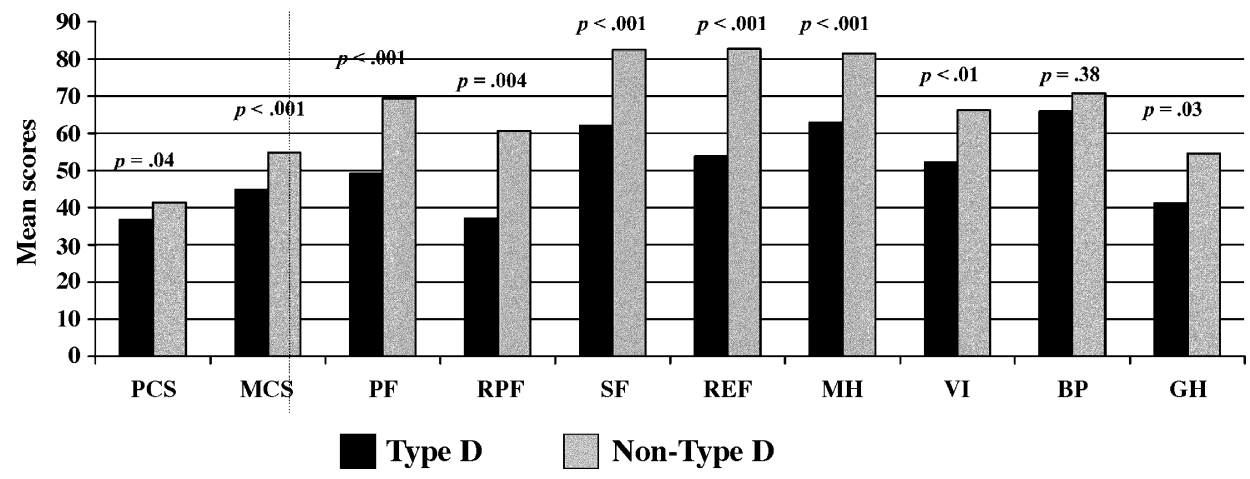

Fig. 1. SF-36 component and subscale scores stratified by Type D personality. PCS = Physical Component summary; MCS=Mental Component summary; $\mathrm{PF}=$ Physical Functioning; RPF = Role Physical Functioning; $\mathrm{SF}=$ Social Functioning; REF = Role Emotional Functioning; MH=Mental Health; VI=Vitality; $\mathrm{BP}=$ Bodily Pain; $\mathrm{GH}=$ General Health.

\section{Statistical analyses}

Discrete variables were compared with the chi-square test (Fischer's exact test when appropriate) and are presented as percentages. Continuous variables were compared with the Student's $t$ test and are presented as means with their corresponding standard deviations (S.D.). Differences between Type D and non-Type D personality on HRQoL were analysed with MANOVA in order to adjust for multiple comparisons. Multivariable logistic regression analyses were used to examine whether Type D was an independent determinant of impaired HRQoL. Prior to analyses, the subscales of the SF-36 were recoded into dichotomous variables, with the lowest tertile of each subscale indicating poor QoL. Dichotomisation has also been advocated by others in order to enhance clinical interpretability [3,21]. In multivariable analyses, we adjusted for all baseline characteristics listed in Table 1. Odds ratios (ORs) with 95\% confidence intervals (CIs) are reported. All tests were two-tailed. A $P$ value $<.05$ was considered statistically significant. All statistical analyses were performed using SPPS version 12.0.1.

\section{Results}

\section{Patients characteristics}

Of the 211 patients, 188 (89\% response rate) completed the questionnaires. Responders and nonresponders did not differ on sex, age, and mean time since transplantation. Given that 2 patients had incomplete data for the Type D scale, analyses were based on 186 patients. The mean time (S.D.) since transplantation for the 186 patients was 7 (5) years.

Of the 186 patients, $18 \%$ met the caseness criteria for Type D personality. Patient characteristics at the time of the survey stratified by Type D personality are presented in Table 1 . Type D patients were more likely to be single $(38 \%$ vs. $15 \% ; P<.01)$ and to have low education ( $47 \%$ vs. $22 \%$; $P<.01)$ compared with non-Type $\mathrm{D}$ patients. No other statistically significant differences were found between the two personality types on characteristics at the time of completion of the questionnaires.

Impact of Type D personality on HRQoL

Type D patients had significantly worse mean scores on the PCS ( 37 vs. $41 ; P=.04$ ) and the MCS (45 vs. 55; $P<.001)$ component scale scores of the SF-36 compared with non-Type D patients (Fig. 1). In addition, except for Bodily Pain, Type D patients had a significantly lower score on all SF-36 subscales compared with non-Type D patients.

When adjusting for demographic and clinical variables, Type D personality remained an independent determinant of impaired PCS (OR, 3.62; 95\% CI, 1.25-10.45) and MCS (OR, 6.13; 95\% CI, 2.23-16.83) (Table 2). Furthermore, Type D personality was an independent determinant of impaired HRQoL on all subscales of the SF-36, except for Bodily Pain and General Health (Table 2). The risk

Table 2

Type D personality as a determinant of impaired HRQoL (adjusted

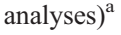

\begin{tabular}{lccc}
\hline & OR & {$[95 \% \mathrm{CI}]$} & $P$ \\
\hline SF-36 component scores & & & \\
PCS & 3.62 & {$[1.25-10.45]$} & $.02^{*}$ \\
MCS & 6.13 & {$[2.23-16.83]$} & $<.01$ \\
& & & \\
SF-36 subscales & & & \\
Physical Functioning & 3.96 & {$[1.47-10.70]$} & $<.01^{* *}$ \\
Role Physical Functioning & 3.52 & {$[1.22-10.15]$} & $.02^{*}$ \\
Social Functioning & 3.58 & {$[1.38-9.27]$} & $<.01^{* *}$ \\
Role Emotional Functioning & 5.17 & {$[1.90-14.07]$} & $<.01^{* *}$ \\
Mental Health & 5.95 & {$[2.14-16.52]$} & $<.01^{* *}$ \\
Vitality & 3.50 & {$[1.24-9.93]$} & $.02^{*}$ \\
Bodily Pain & 1.30 & {$[0.50-3.38]$} & .59 \\
General Health & 1.71 & {$[0.64-4.56]$} & .29 \\
\hline
\end{tabular}

${ }^{a}$ Adjusted for sex, age, time since transplantation, marital status, education, working status, two or more rejection episodes in the first year posttransplantation, need for renal replacement therapy, presence of malignancy, diabetes posttransplantation, and immunosuppressant therapy.

$* P<.05$.

** $P<.01$. 
associated with Type D ranged from $250 \%$ to $513 \%$. Other independent predictors of impaired HRQoL were working status (not working), being in dialysis, two or more rejection episodes in the first year posttransplantation, female sex, and time since diagnosis, depending on the HRQoL subdomain in question (results not shown).

\section{Discussion}

This is the first study to investigate the role of Type D personality as a determinant of impaired HRQoL in heart transplant recipients. We found that patients with a Type D personality reported significantly poorer HRQoL compared with non-Type $\mathrm{D}$ patients. When adjusting for the influence of demographic and clinical characteristics, Type D remained a strong independent determinant of impaired HRQoL, with the risk ranging from more than three- to sixfold.

Type D personality was an independent determinant of impaired HRQoL, with the risk not only being statistically significant but also clinically relevant given the more than three- to sixfold increased risk. These findings concur with those of earlier studies on Type D personality and impaired HRQoL in patients with coronary artery disease [11], chronic heart failure [15], and peripheral arterial disease [16], showing that subgroups of patients with this personality disposition are at increased risk of experiencing poor HRQoL. However, in contrast to previous studies on Type $\mathrm{D}$, only $18 \%$ of the patients in the current study had a Type D personality. This is well below the $24 \%$ to $30 \%$ prevalence rates reported in other studies [11-16]. However, given that Type D has been associated with a four- to eightfold increased risk of mortality [6,11], it is possible that Type D patients rather than non-Type D patients died prior to transplantation. Alternatively, due to a high score on social inhibition Type D patients may present themselves differently to cardiologists than non-Type D patients and voice less of a "fighting spirit," resulting in less of a chance of being listed for transplantation.

The results of the current study should be interpreted with some caution. First, the study was cross-sectional, and patients were not approached at the time of transplantation but at an average of 7 years after transplantation. Therefore, we cannot rule out the possibility that heart transplantation paired with the challenges these patients are faced with, including the tight medical regimen, have influenced scores on the Negative Affectivity and Social Inhibition subscales of the Type D Scale and hence the prevalence of patients with a Type D personality. However, given the higher prevalence of Type $\mathrm{D}$ personality in patients with established CVD, as mentioned previously, and the stability of this personality taxonomy over time, it is more likely that Type D personality is already present prior to transplantation [17]. Second, heart transplant recipients who died before the study were not included.
These patients might have had a poorer HRQoL. However, this is more likely to have led to an underestimation of the effect of Type D on HRQoL rather than an overestimation. Third, although the response rate was high $(89 \%)$, bias could have been introduced from nonresponders. In addition, it is possible that there may be more Type D patients among the nonresponders, although the Social Inhibition component of Type D patients is less likely to have an influence in a "safe" setting, such as completing a questionnaire at home, compared with, e.g., in a faceto-face interview. However, at least nonresponders did not differ on sex, age, and mean time since heart transplantation. Fourth, we used a generic rather than a diseasespecific instrument to assess HRQoL, with disease-specific measures being more sensitive in terms of tapping symptoms pertinent to patients.

Strengths of the current study were the relatively large sample size and response rate of $89 \%$. In addition, the results concur with previous results on Type D personality and HRQoL in patients with coronary artery disease [11], chronic heart failure [15], and peripheral arterial disease [16]. Moreover, they extend our knowledge by showing that Type D personality is of value in heart transplantation research and may be used in clinical practice as a screening tool in order to identify subgroups of heart transplant patients at risk of impaired HRQoL.

In this context, however, it is important to emphasise that having a Type D personality should not be used as a contraindication for transplantation; rather, the purpose of screening would be to identify those patients who require additional support and intervention (e.g., of a psychosocial nature). This may enhance secondary prevention in these high-risk patients and allow Type D patients to benefit from their new heart in terms of HRQoL and prognosis on par with non-Type D patients. A recent study on the efficacy of a multifaceted Internet-based intervention programme designed to enhance the well-being and medical compliance of heart transplant recipients showed a reduction in symptoms of anxiety and depression and an increase in HRQoL social functioning [22]. Type D patients may benefit from a similar intervention, in particular given that they may feel less threatened in sharing their emotions (hence, lowering the Social Inhibition component) in this more impersonal and anonymous setting.

Further research is necessary to understand the impact of Type D personality on the HRQoL of heart transplant recipients. Prospective studies are warranted to investigate the role of Type D personality as a predictor of HRQoL over time and to investigate the prevalence of Type D patients in heart transplant populations at different time points, e.g., when entering the waiting list, around the moment of transplantation, and at regular time points following transplantation. Knowledge of the determinants of poor HRQoL are not only important in order to optimise patient-centered care [2], but poor HRQoL has also been found to predict mortality in patients with CVD [3-5]. 
In conclusion, Type D personality was associated with more than a three- to fivefold increased risk of impaired HRQoL in heart transplant recipients, adjusting for demographic and clinical characteristics. This shows that the Type D personality construct has value in heart transplantation recipients, and that the Type D Scale is a useful screening tool in this distinct patient group to identify subgroups at risk for impaired HRQoL. Prospective studies are now warranted that investigate the impact of Type D personality on HRQoL and clinical prognosis over time.

\section{References}

[1] Taylor DO, Edwards LB, Boucek MM, Keck BM, Hertz MI. Registry of the International Society for Heart and Lung Transplantationtwenty-second official adult heart transplant report-2005. J Heart Lung Transplant 2005;24:956-67.

[2] Krumholz HM, Peterson ED, Ayanian JZ, Chin MH, DeBusk RF, Goldman L, Kiefe CI, Powe NR, Rumsfeld JS, Spertus JA, Weintraub WS. Report of the National Heart, Lung and Blood Institute Working Group on Outcome Research in Cardiovascular Disease. Circulation 2005;111:3158-66.

[3] Spertus JA, Jones P, McDonell M, Fan V, Fihn SD. Health status predicts long-term outcome in outpatients with coronary disease. Circulation 2002;106:43-9.

[4] Soto GE, Jones P, Weintraub WS, Krumholz HM, Spertus JA. Prognostic value of health status in patients with heart failure after acute myocardial infarction. Circulation 2004;110:546-51.

[5] Rodriguez-Artalejo F, Guallar-Castillon G, Pascual CR, Otero CM, Montes AO, Garcia AN, Conthe P, Chiva MO, Banegas JR, Herrera MC. Health-related quality of life as a predictor of hospital readmission and death among patients with heart failure. Arch Intern Med 2005;165:1274-9.

[6] Pedersen SS, Denollet J. Type D personality, cardiac events, and impaired quality of life: a review. Eur J Cardiovasc Prev Rehabil 2003; $10: 241-8$.

[7] Stilley CS, Dew MA, Pilkonis P, Bender A, McNulty M, Christensen A, McCurry KR, Kormos RL. Personality characteristics among cardiothoracic transplant recipients. Gen Hosp Psychiatry 2005;27: $113-8$.

[8] Brandwin M, Trask PC, Schwartz SM, Clifford M. Personality predictors of mortality in cardiac transplant candidates and recipients. J Psychosom Res 2000;49:141-7.

[9] Coffman KL, Brandwin M. The Millon Behavioral Health Inventory life threat reactivity scale as a predictor of mortality in patients awaiting heart transplantation. Psychosomatics 1999;40:44-9.
[10] Shapiro PA, Williams DL, Foray AT, Gelman IS, Wukich N, Sciacca R. Psychosocial evaluation and prediction of compliance problems and morbidity after heart transplantation. Transplantation 1995;60: $1462-6$.

[11] Denollet J, Vaes J, Brutsaert DL. Inadequate response to treatment in coronary heart disease: adverse effects of Type D personality and younger age on 5 year prognosis and quality of life. Circulation 2000;102:630-5.

[12] Pedersen SS, Denollet J. Validity of the Type D personality construct in Danish post MI patients and healthy controls. J Psychosom Res 2004;57:265-72.

[13] Pedersen SS, van Domburg RT, Theuns AMJ, Jordaens L, Erdman RAM. Type D personality is associated with an implantable cardioverter defibrillator and their partners. Psychosom Med 2004;66:714-9.

[14] Pedersen SS, Lemos PA, van Vooren PR, Liu TKK, Daemen J, Erdman RAM, Smits PC, Serruys PWJC, van Domburg RT. Type D personality predicts death or myocardial infarction after bare metal stent or sirolimus-eluting stent implantation. J Am Coll Cardiol 2004;44:997-1001.

[15] Schiffer AA, Pedersen SS, Widdershoven JW, Hendriks EH, Winter JB, Denollet J. The distressed (Type D) personality is independently associated with impaired health status and increased depressive symptoms in chronic heart failure. Eur J Cardiovasc Prev Rehabil 2005; $12: 341-6$.

[16] Aquarius AE, Denollet J, Hamming JF, de Vries J. Role of disease status and Type D personality in outcomes in patients with peripheral arterial disease. Am J Cardiol 2005;96:996-1001.

[17] Denollet J. DS14: Standard assessment of negative affectivity, social inhibition, and Type D personality. Psychosom Med 2005;6: 789-97.

[18] Ware JE, Snow KK, Kosinski M, Gandek B. SF-36 health survey manual and interpretation guide. Boston (Mass): The Health Institute, New England Medical Center, 1993.

[19] Aaronson NK, Muller M, Cohen PDA, Essink-Bot M, Fekkes M, Sanderman R, Sprangers MAG, te Velde A, Verrips E. Translation, validation, and norming of the Dutch language version of the SF-36 Health Survey in community and chronic disease populations. J Clin Epidemiol 1998;51:1055-68.

[20] Ware JE, Kosinski M. Interpreting SF-36 summary health measures: a response. Qual Life Res 2001;10:405-13.

[21] Rumsfeld JS, Magid DJ, Plomondon ME, Sales AE, Grunwald GK, Nathan RE, Spertus JA. History of depression, angina, and quality of life after acute coronary syndromes. Am Heart J 2003; 145:493-9.

[22] Dew MA, Goycoolea JM, Harris RC, Lee A, Zomak R, Dunbar-Jacob J, Rotondi A, Griffith BP, Kormos RL. An Internet-based intervention to improve psychosocial outcomes in heart transplant recipients and family caregivers: development and evaluation. J Heart Lung Transplant 2004;23:745-58. 